\title{
Community-based management and care of people with dementia: A training needs assessment among village health teams in Uganda based on WHO guidelines
}

Christine Karungi ( $\square$ ckarungi@must.ac.ug)

Mbarara University of Science and Technology https://orcid.org/0000-0002-8475-3397

\section{Celestino Obua}

Mbarara University of Science and Technology Faculty of Medicine

Godfrey Z. Rukundo

Mbarara University of Science and Technology Faculty of Medicine

Samuel Maling

Mbarara University of Science and Technology Faculty of Medicine

Jessica E. Haberer

Massachusetts General Hospital center for Global Health and Havard Medical School

Edith K. Wakida

Mbarara University of Science and Technology, Office of Research Administration

Research article

Keywords: Community - based management, Dementia, Training Needs

Posted Date: October 28th, 2019

DOI: https://doi.org/10.21203/rs.2.16517/v1

License: (c) (i) This work is licensed under a Creative Commons Attribution 4.0 International License.

Read Full License 


\section{Abstract}

Background Dementia is a public health problem worldwide with approximately 47 million people living with the disease. Although the symptoms are known and named, dementia remains a hidden problem in low and middle income countries (LMICs) as most people perceive it be a normal aging process. In a bid to improve healthcare, the Uganda government adopted a model of recruiting community-based health assistants known as village health teams (VHTs). These individuals helped in linking the community health units with community members with a goal of promoting health services. However, there is paucity of information about how the VHTs provide community-based management and care for people with dementia and their ability to perform the task. The purpose of this study was to identify the training needs of the VHTs in caring for people with dementia in rural communities of southwestern Uganda.

Methods This study utilized a cross-sectional qualitative design conducted with the help of semistructured interview guide. The WHO guide was adopted in developing interviews on dementia care and management in LMICs.

Results From the study, VHTs reported the need for training with specific to dementia care to equip them with knowledge and skills on early detection and management, care and referral system. Results also show that VHTs were not able to detect dementia early since they perceive it as normal aging process. There was no community engagement in helping people with dementia since most people in the community do not take dementia to be a medical condition. Also there was little support for dementia in these areas as VHTs only referred people with dementia to health facilities in an event of physical ailment. Other organisations like churches only offered prayers only to the aged persons who are very close to the church.

Conclusions The study found that there is an urgent need for training VHTs on caring for individuals with dementia in rural communities of southwestern Uganda. The identified gaps helped in designing the strategies for capacity building through training of VHTs on dementia care in rural communities of southwestern Uganda.

\section{Background}

Dementia is a global public health concern whereby 47 million people are living with this condition (1). This number is expected to triple by the year 2050 among the elderly (2). More than $75 \%$ of these individuals reside in low and middle income countries (LMICs) (3). According to Prince, Comas-Herrera (2) in the World Alzheimer's report (2), the majority of elderly persons with dementia in LMIC have limited access to care and treatment. Additionally, even with diagnosis, the care provided is fragmented, uncoordinated, and unresponsive to the needs of the affected people.

Even when dementia symptoms and signs are known, the illness is given little attention in LMICs as majority of the populace perceive it a normal process of aging $(4,5)$. In Uganda, these individuals do not access healthcare services; nevertheless, aging is among the most common risk factors for developing 
dementia (6-8). As people age, they are likely to experience loss of autonomy and become dependent on significant people around them, particularly relatives and friends; this situation is worse among people with dementia (7). Elderly persons in LMICs with dementia may experience psychological issues, particularly stigma and self-isolation, for which there is limited community engagement and support (9). This situation calls for sensitization as a means to improve care and management for dementia in communities (7).

In 2003, the Uganda government embarked on enrolment of community volunteers who would act as a link between communities and health system $(10,11)$. This programme resulted in the enrolment of Village Health Teams (VHTs) from all villages across the country. VHTs are community volunteers with basic training on the major health programs; they link and connect communities with formal healthcare services (12). They are responsible for conducting home visits, health mobilization and education, management of common illnesses, follow-up of maternal health services, follow-up of discharged patients and those on long-term treatment and community information management (10). They are attached to health centers $(\mathrm{HC})$ at the parish level $(\mathrm{HC} \mathrm{II})$, sub-county level $(\mathrm{HC}$ III), and county level (HC IV) with the mandate to offer community-based preventive and promotive services (13).

There is limited literature on how VHTs are performing in line with dementia care and treatment. However, training programs for healthcare providers in regard to early detection of individuals at risk or in early stages of dementia are important in improving care for these people (14). Engaging VHTs in trainings may help build their capacities in conducting care for dementia in their respective communities (7). This study identified training needs for VHTs in caring for people with dementia in their respective communities.

\section{Methods}

\section{Study design}

This study adopted a cross-sectional qualitative design based on the World Health Organization (WHO) guide for community-based management and care for people with dementia in identifying the training needs of VHTs in rural southwestern Uganda (7).

\section{WHO guide for community workers in LMICs}

Per the WHO, although dementia has no cure or means to alter its progressive course, something can be done to improve care for people with this condition, their caregivers, and families at community level (7). The WHO toolkit was developed to help health assistants in communities in pursuit of promoting health programmes at community level. The tool kit aims at helping health assistants in communities to acquire skills in caring for individuals with dementia: (a) identifying individuals with or those at risk of developing dementia; (b) providing support to people with dementia, their families and caregivers; and (c) sensitizing community members on based practices for improving care of people with dementia in LMICs. 


\section{Study setting and participants}

The study was conducted in Mbarara district which is located in a distance of 270 kilometers (170 miles) by road, southwest of the capital city, Kampala. Mbarara district borders with the districts of Ibanda in the north, Isingiro in the south Sheema in the west, Buhweju in the northwest and Kiruhura in the east (15). Mbarara district is comprised of one municipality (Mbarara Municipality) and two counties (Kashari and Rwampara). It has nineteen sub-counties, from which three sub-counties were selected for inclusion in this study.Participants in the study were VHTs officially recognized by the local council leaders in villages within Bugamba, Ndeija and Rugando sub-counties (16).

\section{Sampling}

Mbarara district was selected as a case study site because it has the highest population of people above sixty years of age $(N=20,442)$ in southwestern Uganda. Similarly, the three selected sub-counties have a high number of people above sixty years of age within the rural settings: Bugamba $(N=1,804)$, Rugando $(N=1,634)$, and Ndeija $(N=1,577)$ in Rwampara county (17). Nyakayojo was excluded from selection because of being in a municipality since our interest for the study was in a rural area. This study therefore focused on Rwampara County in rural southwestern because it has the highest concentration of people aged sixty years and above in Mbarara District and it is a rural community. Rwampara is comprised of four sub-counties, Kashari has six sub-counties, and Mbarara Municipality has six divisions since it an urban setting. In this study, we used fishbowl sampling to choose 10 villages from the three sub-counties, from which one VHT was interviewed per village. We further used fishbowl sampling to select parishes and villages. Gate keepers (local council leaders) to the community were used to select the VHTs as participants in this study.

\section{Procedure}

The lead researcher (CK) conducted in-depth interviews together with three trained research assistants (MN, AK and EK). The three research assistants are graduate students in social sciences. Interviews were conducted in Runyakitara, a local language that is predominantly spoken in this area.

\section{Data collection and tools}

The lead researcher (CK) developed a semi-structured interview guide under the guidance of EKW and $\mathrm{CO}$ following the WHO guidelines on community-based care and management for people with dementia in LMICs (7). The interview guide was comprised of needs assessment, community engagement, early detection and management, and support of people with dementia/family members. The interview guide was translated into Runyakitara and pilot tested in one sub-county that was excluded in the formal study. 
Research assistants transcribed audio-taped data verbatim and the lead researcher (CK) cross checked the transcripts against the audio-taped interviews. Thematic content analysis was adopted (18) with the help of Atlas. Ti version 7 for windows (19). CK and EKW independently read the transcripts and developed codes in line with the emerging themes. The initial coding was done by CK. The coding process was discussed and refined with support from $\mathrm{CO}$ and JEH (senior researchers) and GZR-the mental health specialist on the team.

\section{Results}

\section{Demographics}

In this study, a total of 30 interviews (20 females and 10 males) were conducted. Study participants ages ranged between 18 to 70 years. Most of the participants had attained secondary school education $(n=$ $16)$, whereas the rest had attained primary $(n=9)$ and tertiary education levels $(n=5)$.

\section{Overview}

Results were structured thematically based on the four components shown in the WHO guidelines including: i) Needs assessment; ii) Early detection and management; iii) Community engagement; and iv) Support people with dementia/family members.

\section{Needs assessment}

Regarding needs assessment, most VHTs expressed the need for training as a means to raise awareness on the causes and care for people with dementia. They expressed that this training would help them in their activities of supporting individuals in communities they served.

"What I need is training and nothing else, because I will not lie to you that you give me money and I will give it to them. I need training, I need to be educated so that I will teach them (the patients) to take care of themselves and live for longer." (VHT 1, subcounty3)

In addition, most of the participants specifically highlighted the fact that they needed knowledge on giving support to caregivers of people with dementia. Most of the participants were of the view that dementia, which they confused with forgetfulness, is associated with growing old. On learning of it as a medical condition; they realized a need to be given much more training to enable them to handle and refer people with dementia to health units in case a need arises.

"I need to know what I can teach the caregivers of these people (with dementia) on how they can live with them... like having concrete knowledge and skills because I initially thought it was part of life. Something that happens as people age in life and after knowing it is a disease, where can this person be referred?" (VHT 3, sub county 2) 
When we asked the participants if they were aware about dementia, most participants were able to mention some perceived signs such as aimlessly wandering about or forgetting where their home is, as observed from the quote below:

"Someone can just decide to move but with no specific destination or program (plan) ... and such people are helped by others to find their way back home. Then when a person gets lost and fails to trace their way back home that shows aging because the elderly can somehow get blind when they age." (VHT 5, sub county 1)

Some participants did not think forgetfulness was an indicator for dementia because they related with non-elderly persons having challenges remembering, as expressed in the following quote:

"I think being forgetful is a disease that does not necessarily come with old age because being forgetful has also appeared in middle ages for example I had a friend of mine aged 40-a VHT as well but not elderly but he used to complain that he forgets a lot and finds it difficult to remember even where he would place his phone and to him it was a big problem and he was worried" (VHT 8, sub county 2)

\section{Early detection and management}

Consistent with the expressed limited knowledge of dementia, the majority of participants had difficulty in identifying individuals with dementia particularly among the elderly persons because of their belief that it is normal for elderly persons to forget.

"aah, there are so many things attached (to dementia) and I don't know whether it is a disease or what! But it is a very big problem affecting many people and I do not know how it comes up and I see most elderly people in my village with such a condition but do not know how to help them" (VHT 5, sub county 2)

We, however, found that just like any other illness in the community, the participants used their local knowledge to help people with dementia but with no formal training.

"We always advised them according to our thinking, not that we were taught on how to. So as VHTs, we were visiting them on our own, out of our will and we would advise according to what we think.

Depending on their weakness, we would see how best to advise them. But we weren't trained, but as VHTs, that's what we are supposed to be doing." (VHT 1, sub county 3)

\section{Community engagement}

In terms of community engagement, participants noted that the available community support was largely centered around funeral arrangements. There was no community engagement towards in caring for individuals with dementia. 
"...do you think we really have that (community support)? I do not think so because that would be coming like from social support groups...but still those do not help sick people, but instead in the burial of the deceased." (VHT 10, sub county 3).

Furthermore, the participants acknowledged that there was no direct community support to the aging with signs of dementia because they did not think it was a disease. They had the belief that elderly patients should be referred to their primary care providers to talk to them and make 'simple' prescriptions made them feel better, as illustrated in the quote below:

"There is no direct support for these people since we had not known about it as a disease, but maybe just visiting them and referring them to our "simple" health center Ils to talk to the doctor and some "simple" prescriptions are given and also just the feeling of having talked to the doctor makes them feel better. So that's it. I make sure I give hope to this person, the hope of being better.'” (VHT 3, sub county1)

Participants also noted that there was no support for the elderly in their community unlike neighboring areas where some institutions were extending medical services to the grass root level, as presented in the quote below.

"we have not found any in my village, because they (government) had even registered them to provide some support but they (elderly people) did not get it. No organization has ever come targeting elderly or even those with dementia but in Rugando, I hear that there is some group from Mbarara University that comes and treats the elderly from their homes" (VHT 1, sub county 3)

\section{Support for people with dementia/family members}

In contrast with the lack of community-based support, VHTs reported providing support as a routine part of their practice as VHTs. As usual, they provide support to families in communities since they work as a go-between health centres and people within their respective communities. The support they give to people with dementia is similar to support they would give them in an event of any other medical condition as expressed in the following verbatim.

"I also talk to them (family members/caregivers) and encourage them to stay close to these people (people with dementia) because they are already vulnerable, and they also need feeding. And that they (family members/caregivers) should not leave them (people with dementia) alone and spend long hours without returning. This prevents these people (people with dementia) from being tormented thinking that; my children abandoned me, no one likes to be with me; so to avoid that we encourage them (family members/caregivers) to stay close and understanding to this person." (VHT 5, sub county 1)

"Like I had told you, when I go to visit them (people with dementia) and maybe find them in a dirty environment, unwashed clothes, ... we were told to always help the needy in every possible way. As a VHT member, I ensure they clean up and I help them to improve their hygiene. If I am well quipped for example 
with enough soap, I give them a piece and tell them to take a bathe so that next time I find them clean." (VHT 2, sub county 3)

Furthermore, VHTs communicate with the caregivers about their next day of visit. Participants indicated that the communication was intended to ensure that the caregivers do not miss out talking to the elderly people during their visit.

"I communicate to them when I will be back for the next visit because if you do not, you may go and fail to find them there so, they keep it in mind that VHT said he would be back on this and this day." (VHT 7, sub county 2)

The support from VHTs is present, although it was not specific to dementia and could be informed through better training.

\section{Discussion}

This study assessed training needs among VHTs in southwestern Uganda according to the communitybased management and care tool from the WHO for people with dementia. To our knowledge, this is the first needs assessment for dementia care involving VHTs in Uganda. Our findings indicate that VHTs expressed the need for training on awareness about dementia, skills in early detection and management of dementia, and referral systems. It is of fundamental importance to understand the VHTs' perspective on the need for training to enhance care for individuals with dementia in rural communities of Uganda, because most people in rural communities are known to have poor health seeking behaviors as reported by $\mathrm{CHTs}$ since they act as a linkage between people and health centres in rural communities of Uganda. Equipping VHTs with knowledge and skills may help them in sensitizing individuals with dementia and their caregivers to seek treatment in a timely manner. In addition, VHTs' training may help in promoting community engagement in the care of the elderly persons with dementia in their respective communities. Our findings show that there is limited community support from either, community self-help groups or religious institutions concerning caring for elderly persons with dementia. This is attributed to the fact that majority of the people in these areas have not known dementia as a medical condition; they rather look at it as a normal aging process.

\section{Needs Assessment}

The first step in expanding care capacity for dementia among the elderly is proper training for VHTs. We found familiarity with care for many common physical and mental ailments, yet the VHTs reported the need for training with specific to dementia care to equip them with knowledge and skills on early detection and management, care and referral systems. These findings are consistent with Morgan et al who argues that health providers need to be equipped with capacity and skills in helping people with health needs in our case who elderly individuals with dementia (20). This training is imperative for VHTs who are not professionals in the health field, as this training will equip them with necessary skills in dementia care and management in their respective communities. This knowledge helps in early detection, 
observing people at risk of developing dementia, and proper referral for specialized dementia treatment. Moreover, elderly persons with dementia and their caregivers may be at risk of developing other mental health illness, particularly depression, anxiety and trauma due to burdens they encountered in their lives. In this regard, in an event that VHTs are trained, they can help to link these to health centres for specialized mental healthcare.

Our study found that most VHTs expressed an urgent need for training in support and care for individuals with dementia in their communities. They expressed that training would help them to identify symptoms related to dementia among elderly person and knowledge on when to refer these individuals for specialized medical care. The findings are consistent with those by Saksida and Shantz, who stated that giving training to people volunteering in providing health services facilitates capacity building and improves the way they perform their duties (21). As per the Ugandan health care system, VHTs work on a voluntary basis and available evidence indicates that they have been a success in improving health care at community level (e.g., with immunization and malaria campaigns). This track record implies that when they are given training in handling emerging community related health issues like dementia, VHTs can improve the care of individuals with dementia in communities. The Lancet global mental health series has similarly advocated for task sharing to health volunteers in handling mental health issues as the best promising approach for closing care gaps in mental health care $(22,23)$.

\section{Early detection and management}

This study found that knowledge about dementia is poor among VHTs in Mbarara district. Inadequate knowledge was notable on management of behavioral problems among individuals with dementia and referring them to health units. The majority of the VHTs could not believe that dementia can result into death. Most of the VHTs confused normal forgetting with dementia in which some of them were quoted emphasizing that dementia cuts across all ages, whereby missing appointments among young and middle-aged individuals was dementia. Dementia symptoms are widely considered to be part of normal ageing and not thought to be treatable, especially in rural communities of Uganda. Previous studies identified inadequate knowledge about early signs and symptoms of dementia among health professionals as a barrier to achieving early detection and timely diagnosis of dementia (24). The study by Dreier and Hoffmann on Delphi-MV found that managing people with dementia requires continuous training and refresher courses among healthcare providers for people with dementia $(25,26)$. Relatedly, Nordhus et al, in their study on knowledge about Alzheimer's disease among Norwegian psychologists, reported that there were gaps in promoting training among both professionals and none professionals as a way of promoting care for people with medical conditions in communities (27). In addition, Turner et al, in their study on general practitioners' knowledge, confidence and attitudes in the diagnosis and management of dementia further argues that there is a need to concentrate on risk factors, comorbidities and psychological issues in promoting care for individuals with dementia (28). All these findings point to the fact that the situation of limited awareness on caring for people with dementia particularly the elderly by VHTs in rural Uganda is not an isolated case, but a cross-cutting issue that affects dementia care 
across different societies and individual service providers at different levels of training. The potential impact of training brings hope that VHTs will be able to successfully care for elderly persons with dementia.

\section{Community engagement}

Our results indicate that there is limited community engagement in dementia care and management in communities. We found out that there is limited community engagement from self-help groups, religious institutions and community members in caring for the elderly persons with dementia. This finding was attributed to the fact that majority of the people in communities have not appreciated dementia as a disease, but rather look at it as normal aging process. The little service provided by religious institutions takes the form of routine social support, such as praying and worshipping. While VHTs have been involved in community-based care of individuals with dementia, their services have been limited to routine support that is not informed by dementia-specific training. The findings suggest that VHTs wish to care for patients with dementia, as evidenced by their frequent communication and visits even when they have inadequate knowledge about dementia. Giving them training and education about caring for the patients of dementia will help equip them with necessary skills and knowledge to improve the quality of life of people with dementia in rural communities. This finding is in agreement with prior studies by Dias et al and Guerra et al that found that training health care providers at community level can help in engaging with caregivers and its associated psychological implications $(26,29)$. This implies that, in order to meaningfully support and improve the lives of elderly people with dementia, their caregivers and families, there is need for VHTs to be equipped with knowledge and skills on executing this service in their respective communities (30). Building capacity and empowering VHTs can help in improve access to health care among individuals with dementia.

\section{Support for people with dementia/family members}

Our results further indicate that VHTs provide support to people with dementia through talking to them on issues to do with self-care for healthy ageing, talking to caregivers, making referrals, and supporting family and caregivers. However, this work was not reported as major problem, by virtue of their job description as VHTs provide support to families by linking them to the health system irrespective of which condition is prevailing. The support they provide to the people with dementia and their families is not directly related to dementia rather to the comorbidities of dementia. This implies that VHTs provide support to people with dementia or family members not because they see that these people have dementia but as a general approach of their day to day activities in these communities. Their service lacks a tailored knowledge, which calls for more training so that when they are providing services to these people, they are in position to give the right guidance from an informed point of view. This is consistent with Australian Institute of Health and Welfare Services, which reported that holistic training approaches for healthcare providers are needed to provide improved and sustainable dementia care and management at community level (31). We also found that individuals with dementia and caregivers are likely to have a 
range of needs including health care, transport, and social support that require additional resources from significant people in their respective communities. On the same issue, Weber et al stated that psychosocial support needs to be adopted with other related support given to individuals with individuals in communities (32). In our case, support with psychosocial support is mainly provided by VHTs who conduct routine family visits to communicate to individuals with dementia and caregivers on many matters affecting their lives. What was reported in this study, however, is that though VHTs provide services to the people with dementia, they are not specific in giving the support but as a general support they provide to other people in need, like pregnant mothers and children under five years. This situation calls for a more specific training that will equip these VHTs with knowledge on care and support of individuals with dementia in order to make their work easier and more effective.

\section{Limitations}

Our cross-sectional design with a qualitative approach did not allow the determination of causal relationships in the study variables. This study is also limited by the fact that it was conducted among few stakeholders (VHTs), which could create some gaps in understanding the care for elderly persons with dementia. As such, the findings of this study cannot be generalized to other populations.

\section{Conclusion}

The study found that VHTs in rural southwestern Uganda have training needs in caring for individuals with dementia in their respective communities. Developing a training intervention to address the identified gaps holds great promise toward caring for individuals with dementia in rural communities in Uganda and potentially other similar settings in LMICs.

\section{Declarations}

\section{Abbreviations}

CHWs-Community Health WorkersLMICs-Low- and Middle-Income Countries MADRI - Mbarara Alzheimer's Disease Research InitiativeMUST-REC- Mbarara University Research Ethics CommitteeUNCSTNational Council for Science and TechnologyVHTs-Village Health TeamsWHO -World Health Organization

\section{Acknowledgements}

We would like to thank and acknowledge the contributions of the study participants from the participating communities because we would not have completed the study without them. We recognize the work done by Mariam Nakisekka, Alex Kukundakwe and Esther Kebirungi our Research Assistants. 
We would like to thank the District Health Officer for granting us permission to work with Village Health Teams. This opportunity opened doors for a successful engagement with the community.

\section{Funding}

Research reported in this publication was supported by the Fogarty International Center and the National Institute on Aging of the National Institutes of Health under Award Number D43TW010128. The content is solely the responsibility of the authors and does not necessarily represent the official views of the National Institutes of Health.

\section{Availability of data and materials}

Data on which this manuscript is based will not be publicly available since this work is still ongoing but will be available in future when the intervention component is completed.

\section{Authors' contributions}

All authors contributed to the design of the study, data analysis and writing of the manuscript. The first author (CK) wrote the first draft and the other authors (SM, GZR, JH and EKW) contributed significantly to the revision of the draft. CK and EKW did the data analysis. $\mathrm{CO}$ was the senior researcher on the team for providing overall guidance right from protocol development to the final manuscript. All authors read and approved the final manuscript.

\section{Authors' information}

CK is a Trainee on the on the Mbarara Alzheimer's Disease Research Initiative (MADRI) -(Grant number D43TW010128: Celestino Obua)CO is a senior researcher, a Pharmacologist, and Vice Chancellor of Mbarara University of Science and Technology. He is the Principal Investigator Grant number D43TW010128: Celestino Obua)

GZR is a Senior Lecturer in the Department of Psychiatry, Mbarara University of Science and Technology. $\mathrm{He}$ is also key person on the Mbarara Alzheimer's Disease Research Initiative (MADRI) -(Grant number D43TW010128: Celestino Obua)

Samuel Maling is a senior Lecturer in the Department of Psychiatry, Mbarara University of Science and Technology. He is also key person on the Mbarara Alzheimer's Disease Research Initiative (MADRI)(Grant number D43TW010128: Celestino Obua)

$\mathrm{JH}$ is an Associate Professor at Harvard Medical School and Director of Research at the Massachusetts General Hospital Center for Global Health. She is also key personnel on the Mbarara Alzheimer's Disease Research Initiative (MADRI) -(Grant number D43TW010128: Celestino Obua) 
EKW is a Research Administrator/Manager in the Office of Research Administration at Mbarara University of Science and Technology. She is also key person on the Mbarara Alzheimer's Disease Research Initiative (MADRI) -(Grant number D43TW010128: Celestino Obua)

\section{Ethics approval and consent to participate}

Ethical approval was provided by the Mbarara University Research Ethics Committee under application number 'MUREC 1/7. The study was then registered with the Uganda National Council for Science and Technology under the number 'SS 4937. Permission to conduct interviews in the communities was obtained from the Mbarara District Chief Administrative Officer and the Local Council chairpersons of the villages. All participants gave written informed consent for participation in the study, and for their data to be used solely for study purposes with confidentiality ensured through the use of codes (not names).

Privacy of the participants was ensured by not including identifiable information in addition to conducting the interviews in private spaces. All the audio recorded material and transcripts were securely kept by CK.

\section{Consent for publication}

All participants gave written informed consent for participation in the study, and for their data to be used solely for study purposes with confidentiality ensured through the use of codes (not names).

\section{Competing interests}

The authors declare that they have no competing interests.

\section{References}

1.Sperling RA, Aisen PS, Beckett LA, Bennett DA, Craft S, Fagan AM, et al. Toward defining the preclinical stages of Alzheimer's disease: Recommendations from the National Institute on Aging-Alzheimer's Association workgroups on diagnostic guidelines for Alzheimer's disease. Alzheimer's \& dementia. 2011;7(3):280-92.

2.Prince M, Comas-Herrera A, Knapp M, Guerchet M, Karagiannidou M. World Alzheimer report 2016: improving healthcare for people living with dementia: coverage, quality and costs now and in the future. 2016.

3.Prince $M$, Acosta D, Albanese E, Arizaga R, Ferri CP, Guerra M, et al. Ageing and dementia in low and middle income countries-Using research to engage with public and policy makers. International review of psychiatry. 2008;20(4):332-43. 
4.Cohen L. Toward an anthropology of senility: anger, weakness, and Alzheimer's in Banaras, India. Medical anthropology quarterly. 1995;9(3):314-34.5.Patel V, Prince M. Ageing and mental health in a developing country: who cares? Qualitative studies from Goa, India. Psychological medicine. 2001;31(1):29-38.6.Kyaddondo B. Old Age Mental Health in Uganda. 2015.

7.World Health Organization. Dementia toolkit for community workers in low-and middle-income countries: guide for community-based management and care of people with dementia. Manila: WHO Regional Office for the Western Pacific; 2018.

8.World Health Organization. Dementia Fact Sheet 2017 [cited 2018 10/11/2018]. Available from: http://www.who.int/en/news-room/fact-sheets/detail/dementia.

9.World Health Organization. Global action plan on the public health response to dementia 2017-2025. 2017.

10.Turinawe EB, Rwemisisi JT, Musinguzi LK, de Groot M, Muhangi D, de Vries DH, et al. Selection and performance of village health teams (VHTs) in Uganda: lessons from the natural helper model of health promotion. Human Resources for Health. 2015;13(1):73.

11.Mays DC, O’Neil EJ, Mworozi EA, Lough BJ, Tabb ZJ, Whitlock AE, et al. Supporting and retaining Village Health Teams: an assessment of a community health worker program in two Ugandan districts. International journal for equity in health. 2017;16(1):129.

12.Musinguzi LK, Turinawe EB, Rwemisisi JT, de Vries DH, Mafigiri DK, Muhangi D, et al. Linking communities to formal health care providers through village health teams in rural Uganda: lessons from linking social capital. Human resources for health. 2017;15(1):4.

13.Ministry of Health. Uganda Hospital and Health Centre IV Census Survey 2014. Kampala: 2014. 14.Martín-Carrasco M, Domínguez-Panchón Al, González-Fraile E, Muñoz-Hermoso P, Ballesteros J, Group E. Effectiveness of a psychoeducational intervention group program in the reduction of the burden experienced by caregivers of patients with dementia: the EDUCA-II randomized trial. Alzheimer Disease \& Associated Disorders. 2014;28(1):79-87.

15.Uganda Bureau of Statistics. The National Population and Housing Census 2014- Area Specific Profiles, Kampala, Uganda. 2017.

16.Republic of Uganda. Health: Mbarara District Local Government,; 2017 [cited 2018 6/29/2018]. Available from: http://www.mbarara.go.ug/content/primary-and-early-childhood-learning.

17.Uganda Bureau of Statistics. The National Population and Housing Census 2014-SubCounty Report, Kampala, Uganda. 2016.18.Braun V, Clarke V. Using thematic analysis in psychology. Qualitative research in psychology. 2006;3(2):77-101.19.Muhr T. Atlas. ti: qualitative data analysis, version 7. Berlin: Scientific Software Development GmbH. 2013.

20.Morgan DG, Kosteniuk JG, O'Connell ME, Dal Bello-Haas V, Stewart NJ, Karunanayake C. Dementiarelated work activities of home care nurses and aides: frequency, perceived competence, and continuing 
education priorities. Educational Gerontology. 2016;42(2):120-35.

21.Saksida T, Shantz A, editors. Active management of volunteers: How training and staff support promote commitment of volunteers. Academy of Management Proceedings; 2014: Academy of Management Briarcliff Manor, NY 10510.

22.Jacob K, Sharan P, Mirza I, Garrido-Cumbrera M, Seedat S, Mari JJ, et al. Mental health systems in countries: where are we now? The Lancet. 2007;370(9592):1061-77.23.Saxena S, Thornicroft G, Knapp $\mathrm{M}$, Whiteford $\mathrm{H}$. Resources for mental health: scarcity, inequity, and inefficiency. The lancet. 2007;370(9590):878-89.

24.Brooker D, Fontaine JL, Evans S, Bray J, Saad K. Public health guidance to facilitate timely diagnosis of dementia: ALzheimer's COoperative Valuation in Europe recommendations. International journal of geriatric psychiatry. 2014;29(7):682-93.

25.Dreier A, Hoffmann W. Dementia Care Manager für Patienten mit Demenz. BundesgesundheitsblattGesundheitsforschung-Gesundheitsschutz. 2013;56(10):1398-409.

26.Guerra M, Ferri CP, Fonseca M, Banerjee S, Prince M. Helping carers to care: the 10/66 dementia research group's randomized control trial of a caregiver intervention in Peru. Brazilian Journal of Psychiatry. 2011;33(1):47-54.

27.Nordhus IH, Sivertsen B, Pallesen S. Knowledge about Alzheimer's disease among Norwegian psychologists: The Alzheimer's disease knowledge scale. Aging \& mental health. 2012;16(4):521-8. 28.Turner S, lliffe S, Downs M, Wilcock J, Bryans M, Levin E, et al. General practitioners' knowledge, confidence and attitudes in the diagnosis and management of dementia. Age and ageing. 2004;33(5):461-7.

29.Dias A, Dewey ME, D'Souza J, Dhume R, Motghare DD, Shaji K, et al. The effectiveness of a home care program for supporting caregivers of persons with dementia in developing countries: a randomised controlled trial from Goa, India. PloS one. 2008;3(6):e2333.

30.Dias A. Supporting families to care for people with dementia. Bulletin of the World Health Organization. 2017;95:734-5.31. Health Alo. Dementia in Australia: AlHW; 2012.32.Weber SR, Pirraglia PA, Kunik ME. Use of services by community-dwelling patients with dementia: a systematic review. American Journal of Alzheimer's Disease \& Other Dementias® ${ }^{\circledR}$. 2011;26(3):195-204.Additional file 1 We developed the interview guide specifically for this study. Of note, it followed the WHO guide for community-based management and care of people with dementia (available at https://iris.wpro.who.int/handle/10665.1/14014), which includes the following domains: (i) Needs assessment; ii) Early detection and management; iii) Community engagement; and iv) Support people with dementia/family members. We have uploaded a copy of the interview guide as a supplementary file as directed.

\section{Supplementary Files}


This is a list of supplementary files associated with this preprint. Click to download.

- Additionalfile1.docx 